\title{
The direct and inverse scattering problem for the semilinear Schrödinger equation
}

\author{
Takashi FURUYA
}

\begin{abstract}
We study the direct and inverse scattering problem for the semilinear Schrödinger equation $\Delta u+a(x, u)+k^{2} u=0$ in $\mathbb{R}^{d}$. We show well-posedness in the direct problem for small solutions based on the Banach fixed point theorem, and the solution has the certain asymptotic behavior at infinity. We also show the inverse problem that the semilinear function $a(x, z)$ is uniquely determined from the scattering data. The idea is certain linearization that by using sources with several parameters we differentiate the nonlinear equation with respect to these parameter in order to get the linear one. (see [4, 6, 7].)
\end{abstract}

Key words. inverse scattering problem, semilinear Schrödinger equation, linearization, Herglotz wave function.

\section{Introduction}

In this paper, we study the direct and inverse scattering problem for the semilinear Schrödinger equation

$$
\Delta u+a(x, u)+k^{2} u=0 \text { in } \mathbb{R}^{d},
$$

where $d \geq 2$, and $k>0$. Throughout this paper, we make the following assumptions for the semilinear function $a: \mathbb{R}^{d} \times \mathbb{C} \rightarrow \mathbb{C}$.

Assumption 1.1. We assume that

(i) $a(x, 0)=0$ for all $x \in \mathbb{R}^{d}$.

(ii) $a(x, z)$ is holomorphic at $z=0$ for each $x \in \mathbb{R}^{d}$, that is, there exists $\eta>0$ such that $a(x, z)=\sum_{l=1}^{\infty} \frac{\partial_{z}^{l} a(x, 0)}{l !} z^{l}$ for $|z|<\eta$.

(iii) $\partial_{z}^{l} a(\cdot, 0) \in L^{\infty}\left(\mathbb{R}^{d}\right)$ for all $l \geq 1$. Furthermore, there exists $c_{0}>0$ such that $\left\|\partial_{z}^{l} a(\cdot, 0)\right\|_{L^{\infty}\left(\mathbb{R}^{d}\right)} \leq c_{0}^{l}$ for all $l \geq 1$

(iv) There exists $R>0$ such that $\operatorname{supp}_{z}^{l} a(\cdot, 0) \subset B_{R}$ where $B_{R} \subset \mathbb{R}^{d}$ is a open ball with center 0 and radius $R>0$. 
The above assumptions include the standard type $q(x) u$ where $q \in$ $L^{\infty}\left(\mathbb{R}^{d}\right)$ with compact support, and the power type $q(x) u^{m}$ where $m \in \mathbb{N}$. So far, the inverse problem for the power type in bounded domain via the Dirichlet-to-Neumann map has been studied in [4, 6], and for more general case we refer to [7], which also discusses partial data inverse boundary problem.

We consider the incident field $u_{g}^{i n}$ as the Herglotz wave function

$$
u_{g}^{i n}(x):=\int_{\mathbb{S}^{d-1}} e^{-i k x \cdot \theta} g(\theta) d s(\theta), x \in \mathbb{R}^{d}, g \in L^{2}\left(\mathbb{S}^{d-1}\right),
$$

which solves the free Schrödinger equation $\Delta u_{g}^{i n}+k^{2} u_{g}^{i n}=0$ in $\mathbb{R}^{d}$. The scattered field $u_{g}^{s c}$ corresponding to the incident field $u_{g}^{i n}$ is a solution of the following Schrödinger equation perturbed by the semilinear function $a(x, z)$

$$
\Delta u_{g}+a\left(x, u_{g}\right)+k^{2} u_{g}=0 \text { in } \mathbb{R}^{d},
$$

where $u_{g}$ is total field that is of the form $u_{g}=u_{g}^{s c}+u_{g}^{i n}$, and the scattered field $u^{s c}$ satisfies the Sommerfeld radiation condition

$$
\lim _{r \rightarrow \infty} r^{\frac{d-1}{2}}\left(\frac{\partial u^{s c}}{\partial r}-i k u^{s c}\right)=0
$$

where $r=|x|$.

Since support of the function $a(x, z)$ is compact, the direct scattering problem (1.3)-(1.4) is equivalent to the following integral equation. (see e.g., the argument of Theorem 8.3 in [2].)

$$
u_{g}(x)=u_{g}^{i n}+\int_{\mathbb{R}^{d}} \Phi(x, y) a\left(y, u_{g}(y)\right) d y, \quad x \in \mathbb{R}^{d},
$$

where $\Phi(x, y)$ is the fundamental solution for $-\Delta-k^{2}$ in $\mathbb{R}^{d}$. In the following theorem, we will find a small solution $u_{g}^{s c}$ of (1.5) for small $g \in L^{\infty}\left(\mathbb{R}^{d}\right)$.

Theorem 1.2. We assume that $a(x, z)$ satisfies Assumption 1.1. Then, there exists $\delta_{0} \in(0,1)$ such that for all $\delta \in\left(0, \delta_{0}\right)$ and $g \in L^{\infty}\left(\mathbb{R}^{d}\right)$ with $\|g\|_{L^{\infty}\left(\mathbb{R}^{d}\right)}<\delta^{2}$, there exists a unique solution $u_{g}^{s c} \in L^{\infty}\left(\mathbb{R}^{d}\right)$ with $\left\|u_{g}^{s c}\right\|_{L^{\infty}\left(\mathbb{R}^{d}\right)} \leq \delta$ such that

$$
u_{g}^{s c}(x)=\int_{\mathbb{R}^{d}} \Phi(x, y) a\left(y, u_{g}^{s c}(y)+u_{g}^{i n}(y)\right) d y, x \in \mathbb{R}^{d} .
$$


Theorem 1.2 is proved by the Banach fixed point theorem. By the same argument in Section 19 of [3], the solution $u_{g}^{s c}$ of (1.6) has the following asymptotic behavior

$$
u_{g}^{s c}(x)=C_{d} \frac{\mathrm{e}^{i k r}}{r^{\frac{d-1}{2}}} u_{g}^{\infty}(\hat{x})+O\left(\frac{1}{r^{\frac{d+1}{2}}}\right), r:=|x| \rightarrow \infty, \quad \hat{x}:=\frac{x}{|x|} .
$$

where $C_{d}:=k^{\frac{d-3}{2}} e^{-i \frac{\pi}{4}(d-3)} / 2^{\frac{d+1}{2}} \pi^{\frac{d-1}{2}}$. The function $u_{g}^{\infty}$ is called the scattering amplitude, which is of the form

$$
u_{g}^{\infty}(\hat{x})=\int_{\mathbb{R}^{d}} e^{-i k \hat{x} \cdot y} a\left(y, u_{g}(y)\right) d y, \hat{x} \in \mathbb{S}^{d-1} .
$$

Hence, we are now able to consider the inverse problem to determine the semilinear function $a(x, z)$ from the scattering data $u_{g}^{\infty}(\hat{x})$ for all $g \in$ $L^{2}\left(\mathbb{S}^{d-1}\right)$ with $\|g\|_{L^{2}\left(\mathbb{R}^{d}\right)}<\delta$ where $\delta>0$ is a sufficiently small. We will show the following theorem.

Theorem 1.3. We assume that $a_{j}(x, z)$ satisfies Assumption 1.1. $\quad(j=$ 1,2 .) Let $u_{g, j}^{\infty}$ be the scattering amplitude for the following problem

$$
\begin{gathered}
\Delta u_{j, g}+a_{j}\left(x, u_{j, g}\right)+k^{2} u_{j, g}=0 \text { in } \mathbb{R}^{d}, \\
u_{j, g}=u_{j, g}^{s c}+u_{j, g}^{i n},
\end{gathered}
$$

where $u_{j, g}^{s c}$ satisfies the Sommerfeld radiation (1.4), and $u_{g}^{i n}$ is given by (1.2), and we assume that

$$
u_{1, g}^{\infty}=u_{2, g}^{\infty},
$$

for any $g \in L^{2}\left(\mathbb{S}^{d-1}\right)$ with $\|g\|_{L^{2}\left(\mathbb{R}^{d}\right)}<\delta$ where $\delta>0$ is sufficiently small. Then, we have

$$
a_{1}(x, z)=a_{2}(x, z), x \in \mathbb{R}^{d},|z|<\eta
$$

The idea of the proof is the linearization, which by using sources with several parameters we differentiate the nonlinear equation with respect to these parameter in order to get the linear one. (For such ideas, we refer to [4, 6, 7].) The inverse scattering problems for non-linear Schrödinger equation have been studied in different types of the non-linear potential $a(x, u)$ and in various ways. (See, e.g., [5, 9, 10, 13, 14, 15.) The feature of our works is to recover the whole nonlinearity $a(x, z)$ from the scattering data, that wavenumber $k>0$ is fixed and the incident wave is all of small Herglotz wave functions. 
This paper is organized as follows. In Section 2, we recall the Green function for the Helmholtz equation and its properties. We also prepare the several lemmas required in the forthcoming argument. In Section 3, we prove Theorem 1.2 based on the Banach fixed point theorem. In Section 4, we consider the special solution of (1.3)-(1.4) corresponding to the incident field with several parameters in order to linearize problems. Finally in Section 5, we prove Theorem 1.3.

\section{Preliminary}

First, we recall the Green functions for the Helmholtz equation and its properties. We denote the Green function for $-\Delta-k^{2}$ in $\mathbb{R}^{d}$ by $\Phi(x, y)$, that is, $\Phi(x, y)$ satisfies

$$
\left(-\Delta-k^{2}\right) \Phi(x, y)=\delta(x-y)
$$

for $x, y \in \mathbb{R}^{d}, x \neq y$. In the case of $d=2,3, \Phi(x, y)$ is of the form

$$
\Phi(x, y)= \begin{cases}\frac{i}{4} H_{0}^{(1)}(k|x-y|) & \text { for } x, y \in \mathbb{R}^{2}, x \neq y \\ \frac{e^{i k|x-y|}}{4 \pi|x-y|} & \text { for } x, y \in \mathbb{R}^{3}, x \neq y\end{cases}
$$

Let $q \in L^{\infty}\left(\mathbb{R}^{d}\right)$ with compact support. We denote the Green function for $-\Delta-k^{2}-q$ in $\mathbb{R}^{d}$ by $\Phi_{q}(x, y)$, that is, $\Phi_{q}(x, y)$ satisfies

$$
\left(-\Delta-k^{2}-q\right) \Phi_{q}(x, y)=\delta(x-y) .
$$

for $x, y \in \mathbb{R}^{d}, x \neq y$. It is well known that for every fixed $y, \Phi(x, y)$ and $\Phi_{q}(x, y)$ satisfy the Sommerfeld radiation condition.

We also recall the asymptotics behavior of $\Phi(x, y)$ as $|x| \rightarrow \infty$. In Lemma 19.3 of [3], $\Phi(x, y)$ has the following asymptotics behavior for every fixed $y$,

$$
\Phi(x, y)=C_{d} \frac{\mathrm{e}^{i k|x-y|}}{|x-y|^{\frac{d-1}{2}}}+O\left(\frac{1}{|x-y|^{\frac{d+1}{2}}}\right),|x| \rightarrow \infty
$$

and (see the proof of Theorem 19.5 in [3])

$$
\Phi(x, y)= \begin{cases}O\left(\frac{1}{|x-y|^{d-2}}\right) & d \geq 3, x \neq y \\ O(|\ln | x-y||) & d=2, x \neq y\end{cases}
$$

In Theorem 19.5 of [3], for every $f \in L^{\infty}\left(\mathbb{R}^{d}\right)$ with compact support, $u(x)=$ $\int_{\mathbb{R}^{d}} \Phi(x, y) f(y) d y$ is a unique radiating solution. (that is, $u$ satisfies the 
Sommerfeld radiation condition (1.4).) Furthermore, $u$ has the following asymptotic behavior

$$
u(x)=C_{d} \frac{\mathrm{e}^{i k r}}{r^{\frac{d-1}{2}}} u^{\infty}(\hat{x})+O\left(\frac{1}{r^{\frac{d+1}{2}}}\right), r=|x| \rightarrow \infty, \quad \hat{x}:=\frac{x}{|x|},
$$

where the scattering amplitude $u^{\infty}$ is of the form

$$
u^{\infty}(\hat{x})=\int_{\mathbb{R}^{d}} e^{-i k \hat{x} \cdot y} f(y) d y, \hat{x} \in \mathbb{S}^{d-1} .
$$

The following lemma is given by the same argument as in Lemma 10.4 of [2] or Proposition 2.4 of [8].

Lemma 2.1. Let $q \in L^{\infty}\left(\mathbb{R}^{d}\right)$ with compact support in $B_{R} \subset \mathbb{R}^{d}$ where some $R>0$. We define the Helglotz operator $H: L^{2}\left(\mathbb{S}^{d-1}\right) \rightarrow L^{2}\left(B_{R}(0)\right)$ by

$$
H g(x):=\int_{\mathbb{S}^{d-1}} e^{i k x \cdot \theta} g(\theta) d \theta, x \in B_{R}
$$

and define the operator $T_{q}: L^{2}\left(B_{R}\right) \rightarrow L^{2}\left(B_{R}\right)$ by $T_{q} f:=f+\left.w\right|_{B_{R}(0)}$ where $w$ is a radiating solution such that

$$
\Delta w+k^{2} w+q w=-q f \text { in } \mathbb{R}^{d}
$$

We define the subspace $V$ of $L^{2}\left(B_{R}\right)$ by

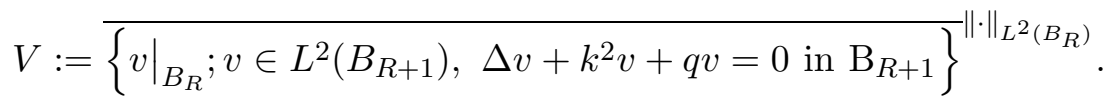

Then, the range of the operator $T_{q} H$ is dense in $V$ with respect to the norm $\|\cdot\|_{L^{2}\left(B_{R}\right)}$, that is,

$$
\overline{T_{q} H\left(L^{2}\left(\mathbb{S}^{d-1}\right)\right)}\|\cdot\|_{L^{2}\left(B_{R}\right)}=V .
$$

The following result is well known. For $d=2$ we refer to [1, and for $d \geq 3$ we refer to [11.

Lemma 2.2. Let $f, q_{1}, q_{2} \in L^{\infty}\left(\mathbb{R}^{d}\right)$ with compact support in $B_{R} \subset \mathbb{R}^{d}$. We assume that

$$
\int_{B_{R}} f v_{1} v_{2} d x=0
$$

for all $v_{1}, v_{2} \in L^{2}\left(B_{R+1}\right)$ with $\Delta v_{j}+k^{2} v_{j}+q_{j} v_{j}=0$ in $B_{R+1} . \quad(j=1,2$.) Then, $f=0$ in $B_{R}$. 


\section{Proof of Theorem 1.2}

In Section 3, we will show Theorem 1.2 based on the Banach fixed point theorem. We denote the Herglotz wave function by

$$
v_{g}(x):=\int_{\mathbb{S}^{d-1}} e^{-i k x \cdot \theta} g(\theta) d s(\theta), x \in \mathbb{R}^{d}, g \in L^{2}\left(\mathbb{S}^{d-1}\right) .
$$

Let $q:=\partial_{z} a(\cdot, 0)$. We define the operator $T: L^{\infty}\left(\mathbb{R}^{d}\right) \rightarrow L^{\infty}\left(\mathbb{R}^{d}\right)$ by

$$
\begin{aligned}
T w(x) & :=\int_{\mathbb{R}^{d}} \Phi_{q}(x, y)\left[a\left(y, w(y)+v_{g}(y)\right)-q(y) w(y)\right] d y \\
& =\int_{\mathbb{R}^{d}} \Phi_{q}(x, y)\left[\sum_{l \geq 2} \frac{\partial_{z}^{l} a(y, 0)}{l !}\left(w(y)+v_{g}(y)\right)^{l}+q(y) v_{g}(y)\right] d y, x \in \mathbb{R}^{d} .
\end{aligned}
$$

Let $X_{\delta}:=\left\{u \in L^{\infty}\left(\mathbb{R}^{d}\right):\|u\|_{L^{\infty}\left(\mathbb{R}^{d}\right)} \leq \delta\right\}$. We remark that $L^{\infty}\left(\mathbb{R}^{d}\right)$ is a Banach space, and $X_{\delta}$ is closed subspace in $L^{\infty}\left(\mathbb{R}^{d}\right)$. To find an unique fixed point of $T$ in $X$, we will show that $T: X_{\delta} \rightarrow X_{\delta}$ and $T$ is a contraction. Let $w \in X_{\delta}$, and let $\delta \in\left(0, \delta_{0}\right)$, and let $\|g\|_{L^{\infty}\left(\mathbb{R}^{d}\right)}<\delta^{2}$. Later, we will choose a appropriate $\delta_{0}>0$.

By $\|g\|_{L^{\infty}\left(\mathbb{R}^{d}\right)}<\delta^{2}$, we have

$$
\left\|v_{g}\right\|_{L^{\infty}\left(\mathbb{R}^{d}\right)} \leq C\|g\|_{L^{\infty}\left(\mathbb{R}^{d}\right)} \leq C \delta^{2}
$$

where $C>0$ is constant only depending on $g$. By (iii) (iv) of Assumption 1.1 , we have

$$
\begin{aligned}
|T w(x)| & \leq \int_{B_{R}}\left|\Phi_{q}(x, y)\right|\left[\sum_{l \geq 2} \frac{c_{0}^{l}}{l !}\left(C_{1} \delta\right)^{l}+C_{1} \delta^{2}\right] d y \\
& \leq C_{2} \delta^{2}\left(\sum_{l \geq 0}\left(C_{1} c_{0} \delta\right)^{l}\right) \int_{B_{R}}\left|\Phi_{q}(x, y)\right| d y
\end{aligned}
$$

where $C_{j}>0(j=1,2)$ is constant independent of $u$ and $\delta$, and so is $\left(\sum_{l \geq 0}\left(C_{1} c_{0} \delta\right)^{l}\right)$ when $\delta>0$ is sufficiently small. Furthermore, by the continuity of difference $\Phi(x, y)-\Phi_{q}(x, y)$ in $x$ and $y$ (see the proof of Theorem 
31.6 in [3]), and the estimation (2.5), we have for $x \in \mathbb{R}^{d}$

$$
\begin{aligned}
\int_{B_{R}}\left|\Phi_{q}(x, y)\right| d y & \leq \int_{B_{R}}\left(|\Phi(x, y)|+\left|\Phi_{q}(x, y)-\Phi(x, y)\right|\right) d y \\
& \leq \int_{B_{R}}\left(|\Phi(x, y)|+C_{3}\right) d y \leq C_{4}
\end{aligned}
$$

which implies that $|T w(x)| \leq C \delta^{2}$ where $C, C_{j}>0(j=3,4)$ is constant independent of $u$ and $\delta$. By choosing $\delta_{0} \in(0,1 / C)$, we conclude that $\|T w\| \leq$ $\delta$, which means $T w \in X_{\delta}$.

Let $w_{1}, w_{2} \in X_{\delta}$. Since we have

$$
\begin{aligned}
& \left(w_{1}(y)+v_{g}(y)\right)^{l}-\left(w_{2}(y)+v_{g}(y)\right)^{l} \\
= & \sum_{m=1}^{l} \frac{l !}{(l-m) ! m !}\left(w_{1}^{m}(y)-w_{2}^{m}(y)\right) v_{g}^{l-m}(y) \\
\leq & \sum_{m=1}^{l} \frac{l !}{(l-m) ! m !}\left(\sum_{h=0}^{m-1} w_{1}^{m-1-h}(y) w_{2}^{h}(y)\right)\left(w_{1}(y)-w_{2}(y)\right) v_{g}^{l-m}(y),
\end{aligned}
$$

and $\left|w_{j}(x)\right| \leq \delta$, then

$$
\begin{aligned}
& \left|T w_{1}(x)-T w_{2}(x)\right| \\
= & \left|\int_{B_{R}} \Phi_{q}(x, y) \sum_{l \geq 2} \frac{\partial_{z}^{l} a(y, 0)}{l !}\left[\left(w_{1}(y)+v_{g}(y)\right)^{l}-\left(w_{2}(y)+v_{g}(y)\right)^{l}\right] d y\right| \\
\leq & \left(\int_{B_{R}}\left|\Phi_{q}(x, y)\right| d y\right) \sum_{l \geq 2} \frac{c_{0}^{l}}{l !} \sum_{m=1}^{l} \frac{l !}{(l-m) ! m !}\left(\sum_{h=0}^{m-1} \delta^{m-1}\right)\left(C_{1}^{\prime} \delta\right)^{l-m}\left\|w_{1}-w_{2}\right\|_{L^{\infty}\left(\mathbb{R}^{d}\right)} \\
\leq & C_{2}^{\prime} \sum_{l \geq 2} \sum_{m=1}^{l} \frac{m}{(l-m) ! m !}\left(c_{0} C_{1}^{\prime} \delta\right)^{l-1}\left\|w_{1}-w_{2}\right\|_{L^{\infty}\left(\mathbb{R}^{d}\right)} \\
\leq & C_{2}^{\prime} \sum_{l \geq 2}\left(\sum_{m=1}^{\infty} \frac{1}{(m-1) !}\right)\left(c_{0} C_{1}^{\prime} \delta\right)^{l-1}\left\|w_{1}-w_{2}\right\|_{L^{\infty}\left(\mathbb{R}^{d}\right)} \\
\leq & C_{3}^{\prime} \sum_{l \geq 2}\left(c_{0} C_{1}^{\prime} \delta\right)^{l-1}\left\|w_{1}-w_{2}\right\|_{L^{\infty}\left(\mathbb{R}^{d}\right)} \\
\leq & C_{3}^{\prime}\left(\sum_{l \geq 0}\left(c_{0} C_{1}^{\prime} \delta\right)^{l}\right) \delta\left\|w_{1}-w_{2}\right\|_{L^{\infty}\left(\mathbb{R}^{d}\right)} \\
\leq & C^{\prime} \delta\left\|u_{1}-u_{2}\right\|_{L^{\infty}\left(\mathbb{R}^{d}\right)}, x \in \mathbb{R}^{d} .
\end{aligned}
$$


where $C^{\prime}, C_{j}^{\prime}>0(j=1,2,3)$ is constant independent of $w_{1}, w_{2}$ and $\delta$. (We remark that $\left(\sum_{l \geq 0}\left(c_{0} C_{1}^{\prime} \delta\right)^{l}\right)$ is also constant when $\delta>0$ is sufficiently small.) By choosing $\delta_{0} \in\left(0,1 / C^{\prime}\right)$, we have $\left\|T w_{1}-T w_{2}\right\|_{L^{\infty}\left(\mathbb{R}^{d}\right)}<$ $\left\|w_{1}-w_{2}\right\|_{L^{\infty}\left(\mathbb{R}^{d}\right)}$. Choosing sufficiently small $\delta_{0} \in\left(0, \min \left(1 / C, 1 / C^{\prime}\right)\right)$ we conclude that $T$ has a unique fixed point in $X_{\delta}$.

Let $w \in X_{\delta}$ be a unique fixed point, that is, $w$ satisfies

$$
w(x)=\int_{\mathbb{R}^{d}} \Phi_{q}(x, y)\left[a\left(y, w(y)+v_{g}(y)\right)-q(y) w(y)\right] d y, x \in \mathbb{R}^{d} .
$$

Since $\Phi_{q}(x, y)$ satisfy the Sommerfeld radiation condition (e.g., see Theorem 31.6 in [3] $), w$ is a radiating solution of $\Delta w+a\left(x, w+v_{g}\right)+k^{2} w=0$ in $\mathbb{R}^{d}$. By the same argument as in Theorem 8.3 of [2], this is equivalent to the integral equation

$$
w(x)=\int_{\mathbb{R}^{d}} \Phi(x, y) a\left(y, w(y)+v_{g}(y)\right) d y, x \in \mathbb{R}^{d},
$$

which means (1.6). Therefore, Theorem 1.2 has been shown.

\section{The special solution}

In Section 4, we consider the special solution of (1.3)-(1.4) corresponding to the incident field with several parameters in order to linearize problems. Let $N \in \mathbb{N}$ be fixed and let $g_{j} \in L^{2}\left(\mathbb{S}^{d-1}\right)$ be fixed $(j=1,2, \ldots, N+1)$. We set

$$
v_{\epsilon}:=\sum_{j=1}^{N+1} \epsilon_{j} \delta^{2} v_{g_{j}}=v_{\left(\delta^{2} \sum_{j=1}^{N+1} \epsilon_{j} g_{j}\right)},
$$

where $v_{g_{j}}$ is the Herglotz wave function defined by (1.2), and $\epsilon_{j} \in(0, \delta)$. Later, we will choose a appropriate $\delta=\delta_{g_{j}, N}>0$. We remark that we can estimate that

$$
\left\|v_{\epsilon}\right\|_{L^{\infty}\left(\mathbb{R}^{d}\right)} \leq C \delta^{2} \sum_{j=1}^{N+1} \epsilon_{j}
$$

where $C>0$ is constant only depending on $g_{j}$. We denote by $\epsilon=\left(\epsilon_{1}, \ldots, \epsilon_{N+1}\right) \epsilon$ $\mathbb{R}^{N+1}$. We will find a small solution $u_{\epsilon}$ of (1.6) that is of the form

$$
u_{\epsilon}=r_{\epsilon}+v_{\epsilon}
$$


This problem is equivalent to

$$
r_{\epsilon}(x)=\int_{\mathbb{R}^{d}} \Phi_{q}(x, y)\left[a\left(y, r_{\epsilon}(y)+v_{\epsilon}(y)\right)-q(y) r_{\epsilon}(y)\right] d y, x \in \mathbb{R}^{d},
$$

where $q:=\partial_{z} a(\cdot, 0)$.

We define the space for $\delta>0$

$$
\tilde{X}_{\delta}:=\left\{r \in L^{\infty}\left(\mathbb{R}^{d} ; C^{N+1}(0, \delta)^{N+1}\right) ; \begin{array}{c}
\operatorname{ess.sup}_{x \in \mathbb{R}^{d}}|r(x, \epsilon)| \leq \sum_{j=1}^{N+1} \epsilon_{j}, \\
\|r\|_{L^{\infty}\left(\mathbb{R}^{d} ; C^{N+1}(0, \delta)^{N+1}\right)} \leq \delta,
\end{array}\right\},
$$

where the norm $\|\cdot\|_{L^{\infty}\left(\mathbb{R}^{d} ; C^{N+1}(0, \delta)^{N+1}\right)}$ is defined by

$$
\|r\|_{L^{\infty}\left(\mathbb{R}^{d} ; C^{N+1}(0, \delta)^{N+1}\right)}:=\sum_{|\alpha| \leq N+1} \sup _{\epsilon \in(0, \delta)^{N+1}} \operatorname{ess} \cdot \sup _{x \in \mathbb{R}^{d}}\left|\partial_{\epsilon}^{\alpha} r(x, \epsilon)\right| .
$$

We remark that $L^{\infty}\left(\mathbb{R}^{d} ; C^{N+1}(0, \delta)^{N+1}\right)$ is a Banach space, and $\tilde{X}_{\delta}$ is closed subspace in $L^{\infty}\left(\mathbb{R}^{d} ; C^{N+1}(0, \delta)^{N+1}\right)$. We will show that following lemma in the same way of Theorem 1.2.

Lemma 4.1. We assume that $a(x, z)$ satisfies Assumption 1.1. Then, there exists $\tilde{\delta}_{0}=\tilde{\delta}_{0, g_{j}, N} \in(0,1)$ such that for all $\delta \in\left(0, \tilde{\delta}_{0}\right)$ there exists an unique solution $r \in \tilde{X}_{\delta}$ such that

$r(x, \epsilon)=\int_{\mathbb{R}^{d}} \Phi_{q}(x, y)\left[a\left(y, r(y, \epsilon)+v_{\epsilon}(y)\right)-q(y) r(y, \epsilon)\right] d y, x \in \mathbb{R}^{d}, \epsilon \in(0, \delta)^{N+1}$.

Proof. We define the operator $\tilde{T}$ from $L^{\infty}\left(\mathbb{R}^{d} ; C^{N+1}(0, \delta)^{N+1}\right)$ into itself by

$$
\begin{aligned}
\tilde{T} r(x, \epsilon) & :=\int_{\mathbb{R}^{d}} \Phi_{q}(x, y)\left[a\left(y, r(y, \epsilon)+v_{\epsilon}(y)\right)-q(y) r(y, \epsilon)\right] d y \\
& =\int_{\mathbb{R}^{d}} \Phi_{q}(x, y)\left[\sum_{l \geq 2} \frac{\partial_{z}^{l} a(y, 0)}{l !}\left(r(y, \epsilon)+v_{\epsilon}(y)\right)^{l}+q(y) v_{\epsilon}(y)\right] d y \\
& =\int_{\mathbb{R}^{d}} \Phi_{q}(x, y)\left[\sum_{l \geq 2} \frac{\partial_{z}^{l} a(y, 0)}{l !} \sum_{m=0}^{l} \frac{l !}{(l-m) ! m !} r^{l-m}(y, \epsilon) v_{\epsilon}^{m}(y)+q(y) v_{\epsilon}(y)\right] d y
\end{aligned}
$$


Let $r \in \tilde{X}_{\delta}$. With (4.2) we have

$$
\begin{aligned}
& |\tilde{T} r(x, \epsilon)| \\
\leq & \left(\int_{B_{R}}\left|\Phi_{q}(x, y)\right| d y\right)\left[\sum_{l \geq 2} c_{0}^{l} \sum_{m=0}^{l} \frac{1}{m !}\left(\sum_{j=1}^{N+1} \epsilon_{j}\right)^{l-m}\left(\tilde{C}_{1} \delta^{2} \sum_{j=1}^{N+1} \epsilon_{j}\right)^{m}+\tilde{C}_{1} \delta^{2} \sum_{j=1}^{N+1} \epsilon_{j}\right] \\
\leq & \tilde{C}_{2}\left[\sum_{l \geq 2} c_{0}^{l}\left(\sum_{m=0}^{\infty} \frac{\tilde{C}_{1}^{m}}{m !}\right)\left(\sum_{j=1}^{N+1} \epsilon_{j}\right)^{l}+\tilde{C}_{1} \delta^{2} \sum_{j=1}^{N+1} \epsilon_{j}\right] \\
\leq & \tilde{C}_{3}\left(\sum_{j=1}^{N+1} \epsilon_{j}\right)^{2} \sum_{l \geq 2} c_{0}^{l}\left(\sum_{j=1}^{N+1} \epsilon_{j}\right)^{l-2}+\tilde{C}_{3} \delta\left(\sum_{j=1}^{N+1} \epsilon_{j}\right) \\
\leq & \tilde{C} \delta\left(\sum_{j=1}^{N+1} \epsilon_{j}\right),
\end{aligned}
$$

where $\tilde{C}, \tilde{C}_{j}>0(j=1,2)$ is constant independent of $r, \delta, \epsilon$ (but, depending on $g_{j}$ and $\left.N\right)$. Furthermore, we consider for $\alpha \in \mathbb{N}^{N+1}$ with $|\alpha| \leq N+1$

$$
\begin{aligned}
& \partial_{\epsilon}^{\alpha} \tilde{T} r(x, \epsilon) \\
= & \int_{\mathbb{R}^{d}} \Phi_{q}(x, y) \partial_{\epsilon}^{\alpha}\left[\sum_{l \geq 2} \frac{\partial_{z}^{l} a(y, 0)}{l !} \sum_{m=0}^{l} \frac{l !}{(l-m) ! m !} r^{l-m}(y, \epsilon) v_{\epsilon}^{m}(y)+q(y) v_{\epsilon}(y)\right] d y .
\end{aligned}
$$

Since $\left|\partial_{\epsilon_{j}} v_{\epsilon}(x)\right| \leq \tilde{C}_{1}^{\prime} \delta^{2}$ and $\left|\partial_{\epsilon}^{\alpha} r^{l-m}(x, \epsilon) v_{\epsilon}^{m}(x)\right| \leq \tilde{C}_{2}^{\prime}(l-m) ! m ! \delta^{l-m}\left(\tilde{C}_{2}^{\prime} \delta^{2}\right)^{m}$, we have

$$
\begin{aligned}
\left|\partial_{\epsilon}^{\alpha} \tilde{T} r(x, \epsilon)\right| & \leq\left(\int_{B_{R}}\left|\Phi_{q}(x, y)\right| d y\right)\left[\sum_{l \geq 2} \frac{c_{0}^{l}}{l !} \sum_{m=0}^{l} \frac{l ! m !(l-m) !}{(l-m) ! m !} \delta^{l+m}\left(\tilde{C}_{2}^{\prime}\right)^{m}+\tilde{C}_{3}^{\prime} \delta^{2}\right] \\
& \leq \tilde{C}_{4}^{\prime} \delta^{2}\left(\sum_{l \geq 2}\left(c_{0} \delta\right)^{(l-2)} \sum_{m=0}^{\infty}\left(\tilde{C}_{2}^{\prime} \delta\right)^{m}\right)+\tilde{C}_{4}^{\prime} \delta^{2} \leq \tilde{C}_{5}^{\prime} \delta^{2}
\end{aligned}
$$

where $\tilde{C}_{j}^{\prime}>0(j=3,4,5)$ is also constant independent of $r, \delta, \epsilon$ (but depending on $\alpha$ ). Then, we have

$$
\sum_{|\alpha| \leq N+1} \sup _{\epsilon \in(0, \delta)^{N+1}} \operatorname{ess.sup} \operatorname{su\in }_{x \in \mathbb{R}^{d}}\left|\partial_{\epsilon}^{\alpha} \tilde{T} r(x, \epsilon)\right| \leq \tilde{C}^{\prime} \delta^{2}
$$


where $\tilde{C}^{\prime}$ is constant independent of $r, \delta, \epsilon$. (depending on $g_{j}$ and $N$.) By choosing $\tilde{\delta}_{0} \in\left(0, \min \left(1 / \tilde{C}, 1 / \tilde{C}^{\prime}\right)\right)$, we conclude that $\tilde{T} r \in \tilde{X}_{\delta}$.

Let $r_{1}, r_{2} \in \tilde{X}_{\delta}$. By similar argument in (3.6) we have

$$
\begin{aligned}
& \tilde{T} r_{1}(x, \epsilon)-\tilde{T} r_{2}(x, \epsilon) \\
= & \int_{B_{R}} \Phi_{q}(x, y) \sum_{l \geq 2} \frac{\partial_{z}^{l} a(y, 0)}{l !}\left[\left(r_{1}(y, \epsilon)+v_{\epsilon}(y)\right)^{l}-\left(r_{2}(y, \epsilon)+v_{\epsilon}(y)\right)^{l}\right] d y \\
= & \int_{B_{R}} \Phi_{q}(x, y) \sum_{l \geq 2} \frac{\partial_{z}^{l} a(y, 0)}{l !} \sum_{m=1}^{l} \frac{l !}{(l-m) ! m !} v_{\epsilon}^{l-m}(y) \\
& \times \sum_{h=0}^{m-1} r_{1}^{m-1-h}(y, \epsilon) r_{2}^{h}(y, \epsilon)\left(r_{1}(y, \epsilon)-r_{2}(y, \epsilon)\right) d y .
\end{aligned}
$$

Then, we have for $\alpha \in \mathbb{N}^{N+1}$ with $|\alpha| \leq N+1$

$$
\begin{aligned}
& \left|\partial_{\epsilon}^{\alpha}\left(\tilde{T} r_{1}(x)-\tilde{T} r_{2}(x)\right)\right| \\
\leq & \int_{B_{R}}\left|\Phi_{q}(x, y)\right| \sum_{\beta \leq \alpha} \frac{\alpha !}{(\alpha-\beta) ! \beta !} \sum_{l \geq 2} \frac{\left|\partial_{z}^{l} a(y, 0)\right|}{l !} \sum_{m=1}^{l} \frac{l !}{(l-m) ! m !} \\
\times & \sum_{h=0}^{m-1}\left|\partial_{\epsilon}^{\beta}\left(v_{\epsilon}^{l-m}(y) r_{1}^{m-1-h}(y, \epsilon) r_{2}^{h}(y, \epsilon)\right)\right|\left|\partial_{\epsilon}^{\alpha-\beta}\left(r_{1}(y, \epsilon)-r_{2}(y, \epsilon)\right)\right| d y .
\end{aligned}
$$

Since

$$
\left|\partial_{\epsilon}^{\beta}\left(v_{\epsilon}^{l-m}(y) r_{1}^{m-1-h}(y, \epsilon) r_{2}^{h}(y, \epsilon)\right)\right| \leq \tilde{C}_{1}^{\prime \prime}(l-m) !(m-1-h) ! h !\left(\tilde{C}_{1}^{\prime \prime} \delta^{2}\right)^{l-m} \delta^{m-1-h} \delta^{h},
$$

where $\tilde{C}_{1}^{\prime \prime}$ is constant independent of $r_{1}, r_{2}$ and $\delta$ (depending on $\beta$ ), we have 
that

$$
\begin{aligned}
& \left|\partial_{\epsilon}^{\alpha}\left(\tilde{T} r_{1}(x)-\tilde{T} r_{2}(x)\right)\right| \\
\leq & \tilde{C}_{2}^{\prime \prime}\left(\sum_{\beta \leq \alpha} \frac{\alpha !}{(\alpha-\beta) ! \beta !} \sum_{l \geq 2} \frac{c_{0}^{l}}{l !} \sum_{m=1}^{l} \sum_{h=0}^{m-1} \frac{l !(l-m) !(m-1-h) ! h !}{(l-m) ! m !} \delta^{2 l-m-1}\left(\tilde{C}_{1}^{\prime \prime}\right)^{l-m}\right)\left\|r_{1}-r_{2}\right\| \\
\leq & \tilde{C}_{3}^{\prime \prime} \delta\left(\sum_{l \geq 2}\left(c_{0} \delta\right)^{l-2} \sum_{m=1}^{l}\left(\tilde{C}_{1}^{\prime \prime} \delta\right)^{l-m} \sum_{h=0}^{m-1} \frac{(m-1-h) ! h !}{m !}\right)\left\|r_{1}-r_{2}\right\| \\
\leq & \tilde{C}_{4}^{\prime \prime} \delta\left(\sum_{l \geq 2}\left(c_{0} \delta\right)^{l-2} \sum_{p=0}^{\infty}\left(\tilde{C}_{1}^{\prime \prime} \delta\right)^{p}\right)\left\|r_{1}-r_{2}\right\| \leq \tilde{C}_{5}^{\prime \prime} \delta\left\|r_{1}-r_{2}\right\|_{L^{\infty}\left(\mathbb{R}^{d} ; C^{N+1}(0, \delta)^{N+1}\right)},
\end{aligned}
$$

which implies that

$$
\sum_{|\alpha| \leq N+1} \sup _{\epsilon \in(0, \delta)^{N+1}} \operatorname{ess}_{\sup } \sup _{x \in \mathbb{R}^{d}}\left|\partial_{\epsilon}^{\alpha}\left(\tilde{T} r_{1}(x, \epsilon)-\tilde{T} r_{2}(x, \epsilon)\right)\right| \leq \tilde{C}^{\prime \prime} \delta\left\|r_{1}-r_{2}\right\|
$$

where $\tilde{C}_{j}^{\prime \prime}, \tilde{C}^{\prime \prime}>0(j=2,3,4)$ is constant independent of $r_{1}, r_{2}$ and $\delta$. By choosing $\tilde{\delta}_{0} \in\left(0, \min \left(1 / \tilde{C}, 1 / \tilde{C}^{\prime}, 1 / \tilde{C}^{\prime \prime}\right)\right)$, we have $\left\|T r_{1}-\operatorname{Tr}_{2}\right\|<\left\|r_{1}-r_{2}\right\|$, which implies that $\tilde{T}$ has a unique fixed point in $\tilde{X}_{\delta}$. Lemma 4.1 has been shown.

\section{$5 \quad$ Proof of Theorem 1.3}

In Section 5, we will show Theorem 1.3. Since $a(x, z)$ is holomorphic at $z=0$ by (ii) of Assumption 1.1, it is sufficient to show that

$$
\partial_{z}^{l} a_{1}(x, 0)=\partial_{z}^{l} a_{2}(x, 0), x \in \mathbb{R}^{d},
$$

for all $l \in \mathbb{N}$. Let $N \in \mathbb{N}$ and let $g_{j} \in L^{2}\left(\mathbb{S}^{d-1}\right)(j=1,2, \ldots, N+1)$. Let $\delta \in\left(0, \min \left(\delta_{0}, \tilde{\delta}_{0}\right)\right)$ be chosen as sufficiently small and depending on $N$ and $g_{j} .\left(\delta_{0}, \tilde{\delta}_{0}\right.$ are corresponding to Theorem 1.2 and Lemma 4.1, respectively.) From Section 4, we obtain the unique solution $r_{\epsilon, j} \in \tilde{X}_{\delta}(j=1,2)$ such that

$$
\Delta r_{\epsilon, j}+a_{j}\left(x, r_{\epsilon, j}+v_{\epsilon}\right)+k^{2} r_{\epsilon, j}=0 \text { in } \mathbb{R}^{d},
$$

where $r_{\epsilon, j}$ satisfies the Sommerfeld radiation, and $v_{\epsilon}$ is given by (4.1). The solution $r_{\epsilon, j}$ has the form

$$
r_{\epsilon, j}(x)=\int_{\mathbb{R}^{d}} \Phi(x, y) a_{j}\left(y, r_{\epsilon, j}(y)+v_{\epsilon}(y)\right) d y, x \in \mathbb{R}^{d}, \epsilon \in(0, \delta)^{N+1} .
$$


By the assumption of Theorem 1.3 we have

$$
r_{\epsilon, 1}^{\infty}(\hat{x})=r_{\epsilon, 2}^{\infty}(\hat{x}), \hat{x} \in \mathbb{S}^{d-1}, \epsilon \in(0, \delta)^{N+1},
$$

where $r_{\epsilon, j}^{\infty}$ is a scattering amplitude for $r_{\epsilon, j}$, and it has the form

$$
r_{\epsilon, j}^{\infty}(\hat{x})=\int_{\mathbb{R}^{d}} e^{-i k \hat{x} \cdot y} a_{j}\left(y, r_{\epsilon, j}(y)+v_{\epsilon}(y)\right) d y, \hat{x} \in \mathbb{S}^{d-1}, \epsilon \in(0, \delta)^{N+1} .
$$

In order to linearize (5.3), we will differentiate it with respect to $\epsilon_{l}$ ( $l=$ $1, \ldots, N+1$ ), which is possible because $r_{\epsilon, j} \in \tilde{X}_{\delta}$. Then, we have

$$
\partial_{\epsilon_{l}} r_{\epsilon, j}(x)=\int_{\mathbb{R}^{d}} \Phi(x, y) \partial_{z} a_{j}\left(y, r_{\epsilon, j}(y)+v_{\epsilon}(y)\right)\left(\partial_{\epsilon_{l}} r_{\epsilon, j}(y)+\delta^{2} v_{g_{l}}(y)\right) d y .
$$

As $\epsilon \rightarrow+0$ we have by setting $q_{j}:=\partial_{z} a_{j}(y, 0)$

$$
w_{l, j}(x):=\left.\partial_{\epsilon_{l}} r_{\epsilon, j}\right|_{\epsilon=0}(x)=\int_{\mathbb{R}^{d}} \Phi(x, y) q_{j}(y)\left(w_{l, j}(y)+\delta^{2} v_{g_{l}}(y)\right) d y,
$$

which implies that

$$
\Delta w_{l, j}+k^{2} w_{l, j}=-q_{j}\left(w_{l, j}+\delta^{2} v_{g_{l}}\right) \text { in } \mathbb{R}^{d} .
$$

By setting $u_{l, j}:=w_{l, j}+\delta^{2} v_{g_{l}}$ we have

$$
\Delta u_{l, j}+k^{2} u_{l, j}+q_{j} u_{l, j}=0 \text { in } \mathbb{R}^{d}
$$

By setting $u_{l}:=u_{l, 1}-u_{l, 2}\left(=w_{l, 1}-w_{l, 2}\right)$ we have

$$
\Delta u_{l}+k^{2} u_{l}+q_{1} u_{l}=\left(q_{2}-q_{1}\right) u_{l, 2} \text { in } \mathbb{R}^{d},
$$

and we also have

$$
\left(q_{2}-q_{1}\right) u_{h, 1} u_{l, 2}=u_{h, 1} \Delta u_{l}-u_{l} \Delta u_{h, 1} \text { in } \mathbb{R}^{d} .
$$

Differentiating (5.4) with respect to $\epsilon_{l}$ and as $\epsilon \rightarrow 0$ we have

$$
\int_{\mathbb{R}^{d}} e^{-i k \hat{x} \cdot y} q_{1}(y)\left(w_{l, 1}(y)+\delta^{2} v_{g_{l}}(y)\right) d y=\int_{\mathbb{R}^{d}} e^{-i k \hat{x} \cdot y} q_{2}(y)\left(w_{l, 2}(y)+\delta^{2} v_{g_{l}}(y)\right) d y,
$$

which means that $w_{l, 1}^{\infty}=w_{l, 2}^{\infty}$, where $w_{l, j}^{\infty}$ is a scattering amplitude of $w_{l, j}$. By setting $\hat{w}_{l}:=w_{l, 1}-w_{l, 2}$ we have

$$
\Delta \hat{w}_{l}+k^{2} \hat{w}_{l}=0 \text { in } \mathbb{R} \backslash \overline{B_{R}},
$$


where $\hat{w}_{l}$ satisfies the Sommerfeld radiation condition, and the scattering amplitude $\hat{w}_{l}^{\infty}$ of $\hat{w}_{l}$ vanishes. Then, we have $\hat{w}_{l}=0$ (that is, $u_{l}=0$ ) in $\mathbb{R} \backslash \overline{B_{R}}$, which implies that by the Green's second theorem we have $(l, h=$ $1, \ldots, N+1)$

$$
\begin{aligned}
0 & =\int_{\partial B_{R+1}} u_{h, 1} \partial_{\nu} u_{l}-u_{l} \partial_{\nu} u_{h, 1} d s \\
& =\int_{B_{R+1}} u_{h, 1} \Delta u_{l}-u_{l} \Delta u_{h, 1} d x \\
& =\int_{B_{R}}\left(q_{2}-q_{1}\right) u_{h, 1} u_{l, 2} d x .
\end{aligned}
$$

By (5.8), and definition of $H$ and $T_{q_{j}}$ in Section $2, u_{l, j}$ can be of the form

$$
u_{l, j}=\delta^{2} T_{q_{j}} H g_{l},
$$

and dividing by $\delta^{4}>0$,

$$
0=\int_{B_{R}}\left(q_{2}-q_{1}\right) T_{q_{1}} H g_{h} T_{q_{2}} H g_{l} d x
$$

Combining Lemma 2.1 with Lemma 2.2, we conclude that $q_{1}=q_{2}$.

By induction, we will show (5.1). In the first part of this section, the case of $l=1$ has been shown. We assume that

$$
\partial_{z}^{l} a_{1}(x, 0)=\partial_{z}^{l} a_{2}(x, 0)
$$

for all $l=1,2, \ldots, N$. We will show the case of $l=N+1$. We alredy have shown that $q_{1}=q_{2}$ and $w_{l, 1}^{\infty}=w_{l, 2}^{\infty}$, which implies that by the uniqueness of the linear Schrödinger equation (5.8) we have

$$
w_{l, 1}=w_{l, 2} \text { in } \mathbb{R}^{d}
$$

for all $l=1, \ldots, N+1$.

We set $q:=q_{1}=q_{2}$ and $w_{l}:=w_{l, 1}=w_{l, 2}$. By subinduction we will show that for all $h \in \mathbb{N}$ with $1 \leq h \leq N$

$$
\left.\partial_{\epsilon_{l_{1} \ldots \epsilon_{l}}^{h}}^{h} r_{\epsilon, 1}\right|_{\epsilon=0}=\left.\partial_{\epsilon_{l_{1} \ldots \epsilon_{l}}}^{h} r_{\epsilon, 2}\right|_{\epsilon=0},
$$

where $l_{1}, \ldots l_{h} \in\{1, \ldots, N+1\}$. We already have shown that (5.19) holds for $h=1$. We assume that (5.19) holds for all $h \leq K \leq N-1$. (If $N=1$, this 
subinduction is skipped.) By differentiating (5.3) with respect to $\partial_{\epsilon_{l_{1}} \ldots \epsilon_{l_{K+1}}^{K+1}}$ we have

$$
\begin{aligned}
\partial_{\epsilon_{l_{1} \ldots \epsilon_{K+1}}}^{K+1} r_{\epsilon, j}(x)= & \int_{\mathbb{R}^{d}} \Phi(x, y)\left\{\partial_{z}^{K+1} a_{j}\left(y, r_{\epsilon, j}(y)+v_{\epsilon}(y)\right) \prod_{h=1}^{K+1}\left(\partial_{\epsilon_{l_{h}}} r_{\epsilon, j}(y)+\delta^{2} v_{g_{l_{h}}}(y)\right)\right. \\
& \left.+\partial_{z} a_{j}\left(y, r_{\epsilon, j}(y)+v_{\epsilon}(y)\right) \partial_{\epsilon_{l_{1} \ldots \epsilon_{K+1}}^{K+1}} r_{\epsilon, j}(y)+R_{K, j}(y, \epsilon)\right\} d y, \quad \text { (5.20) }
\end{aligned}
$$

where $R_{K, j}(y, \epsilon)$ is a polynomial of $\partial_{z}^{h} a_{j}\left(y, r_{\epsilon, j}(y)+v_{\epsilon}(y)\right)$ and $\partial_{\epsilon_{l_{1}} \ldots \epsilon_{l_{h}}}^{h}\left(r_{\epsilon, j}(y)+v_{\epsilon}(y)\right)$ for $1 \leq h \leq K$. As $\epsilon \rightarrow 0$ we have

$$
\begin{aligned}
& \left.\partial_{\epsilon_{l_{1}} \ldots \epsilon_{l_{K+1}}}^{K+1} r_{\epsilon, j}\right|_{\epsilon=0}(x)=\int_{\mathbb{R}^{d}} \Phi(x, y)\left\{\partial_{z}^{K+1} a_{j}(y, 0) \prod_{h=1}^{K+1}\left(w_{l_{h}}(y)+\delta^{2} v_{g_{l_{h}}}(y)\right)\right. \\
& \left.+\left.q(y) \partial_{\epsilon_{l_{1} \ldots \epsilon_{K+1}}^{K+1}}^{K+1} r_{\epsilon, j}\right|_{\epsilon=0}(y)+R_{K, j}(y, 0)\right\} d y .
\end{aligned}
$$

We set $\tilde{w}_{K+1, j}:=\left.\partial_{\epsilon_{l_{1}} \ldots \epsilon_{l} K+1}^{K+1} r_{\epsilon, j}\right|_{\epsilon=0}$ and set $\tilde{w}_{K+1}:=\tilde{w}_{K+1,1}-\tilde{w}_{K+1,2}$. By assumptions of induction and subinduction we have $R_{K, 1}(y, 0)=R_{K, 2}(y, 0)$ and $\partial_{z}^{K+1} a_{1}(\cdot, 0)=\partial_{z}^{K+1} a_{2}(\cdot, 0)$, which implies that

$$
\tilde{w}_{K+1}(x)=\int_{\mathbb{R}^{d}} \Phi(x, y) q(y) \tilde{w}_{K+1}(y) d y,
$$

which is equivalent to

$$
\Delta \tilde{w}_{K+1}+k^{2} \tilde{w}_{K+1}+q \tilde{w}_{K+1}=0 \text { in } \mathbb{R}^{d},
$$

where $\tilde{w}_{K+1}$ satisfies Sommerfeld radiation condition. By differentiating (5.4) with respect to $\partial_{\epsilon_{l_{1}} \ldots \epsilon_{l_{K+1}}}^{K+1}$ and as $\epsilon \rightarrow 0$ we have

$$
\tilde{w}_{K+1,1}^{\infty}=\tilde{w}_{K+1,2}^{\infty}
$$

where $\tilde{w}_{K+1, j}^{\infty}$ is a scattering amplitude of $\tilde{w}_{K+1, j}$. (5.24) means that $\tilde{w}_{K+1}^{\infty}=$ 0 , which implies that by Rellich theorem, we conclude that $\tilde{w}_{K+1}=0$ in $\mathbb{R}^{d}$. (5.19) for the case of $K+1$ has been shown, and the claim (5.19) holds for all $h=1, \ldots, N$ by subinduction.

By differentiating (5.3) with respect to $\partial_{\epsilon_{1} \ldots \epsilon_{K+1}}^{N+1}$, and as $\epsilon \rightarrow 0$ (the same argument in (5.20)-(5.22) ) we have

$$
\tilde{w}_{N+1}(x)=\int_{\mathbb{R}^{d}} \Phi(x, y)\left\{\left(\partial_{z}^{N+1} a_{1}(x, 0)-\partial_{z}^{N+1} a_{2}(x, 0)\right) \prod_{h=1}^{N+1}\left(w_{h}(y)+\delta^{2} v_{g_{h}}(y)\right)\right.
$$




$$
\left.+q(y) \tilde{w}_{N+1}(y)\right\} d y
$$

where $\tilde{w}_{N+1, j}:=\left.\partial_{\epsilon_{1} \ldots \epsilon_{N+1}}^{N+1} r_{\epsilon, j}\right|_{\epsilon=0}$ and set $\tilde{w}_{N+1}:=\tilde{w}_{N+1,1}-\tilde{w}_{N+1,2}$. This is equivalent to

$$
\Delta \tilde{w}_{N+1}+k^{2} \tilde{w}_{N+1}+q \tilde{w}_{N+1}=-f \prod_{h=1}^{N+1} \delta^{2} T_{q} H g_{h} \text { in } \mathbb{R}^{d},
$$

where $f(x):=\partial_{z}^{N+1} a_{1}(x, 0)-\partial_{z}^{N+1} a_{2}(x, 0)$. By differentiating (5.4) with respect to $\partial_{\epsilon_{1} \ldots \epsilon_{K+1}}^{N+1}$ and as $\epsilon \rightarrow 0$ (the same argument in (5.24) ) we have

$$
\tilde{w}_{N+1}^{\infty}=0,
$$

where $\tilde{w}_{N+1}^{\infty}$ is a scattering amplitude of $\tilde{w}_{N+1}$. Then, we have $\tilde{w}_{N+1}=0$ in $\mathbb{R} \backslash \overline{B_{R}}$.

Let $\tilde{v} \in L^{2}\left(B_{R+1}\right)$ be a solution of $\Delta \tilde{v}+k^{2} \tilde{v}+q \tilde{v}=0$ in $B_{R+1}$. By the Green's second theorem and (5.26) we have

$$
\begin{aligned}
0 & =\int_{\partial B_{R+1}} \tilde{v} \partial_{\nu} \tilde{w}_{N+1}-\tilde{v} \partial_{\nu} \tilde{w}_{N+1} d s \\
& =\int_{B_{R+1}} \tilde{v} \Delta \tilde{w}_{N+1}-\tilde{w}_{N+1} \Delta \tilde{v} d x \\
& =\int_{B_{R+1}}-f \prod_{h=1}^{N+1} \delta^{2} T_{q} H g_{h} \tilde{v} d x
\end{aligned}
$$

which implies that dividing by $\delta^{2}>0$

$$
\int_{B_{R+1}} f \prod_{h=1}^{N+1} T_{q} H g_{h} \tilde{v} d x=0
$$

Let $v \in L^{2}\left(B_{R+1}\right)$ be a solution of $\Delta v+k^{2} v+q v=0$ in $B_{R+1}$. By Lemma 2.1 we can choose $g_{N+1}$ as $g_{N+1, j} \in L^{2}\left(B_{R+1}\right)$ such that $T_{q} H g_{N+1, j} \rightarrow v$ in $L^{2}\left(B_{R}\right)$ as $j \rightarrow \infty$. Then, we have that

$$
\int_{B_{R+1}} f \prod_{h=1}^{N} T_{q} H g_{h} v \tilde{v} d x=0 .
$$

which implies that by Lemma 2.2

$$
f \prod_{h=1}^{N} T_{q} H g_{h}=0
$$


By Theorem 5.1 of [12, we can choose a solution $u_{h} \in L^{2}\left(B_{R+1}\right)(h=$ $1, \ldots, N)$ of $\Delta u_{h}+k^{2} u_{h}+q u_{h}=0$ in $B_{R+1}$, which is of the form

$$
u_{h}(x)=e^{x \cdot p_{h}}\left(1+\psi_{h}\left(x, p_{h}\right)\right),
$$

with $\left\|\psi_{h}\left(\cdot, p_{h}\right)\right\|_{L^{2}\left(B_{R+1}\right)} \leq \frac{C}{\left|p_{h}\right|}$ where $C>0$ is a constant, and $p_{h}=a_{h}+i b_{h}$, $a_{h}, b_{h} \in \mathbb{R}^{d}$ such that $\left|a_{h}\right|=\left|b_{h}\right|$ and $a_{h} \cdot b_{h}=0$ (which implies that $p_{h} \cdot p_{h}=$ $0)$, and $a_{h} \neq a_{h^{\prime}}, b_{h} \neq b_{h^{\prime}}$.

Multiplying (5.31) by $\bar{f} \prod_{h=1}^{N+1} e^{-x \cdot p_{h}}$ we have

$$
|f|^{2} \prod_{h=1}^{N} e^{-x \cdot p_{h}} T_{q} H g_{h}=0
$$

which implies that

$$
\int_{B_{R}}|f|^{2}\left(\prod_{h=1}^{N-1} e^{-x \cdot p_{h}} T_{q} H g_{h}\right) e^{-x \cdot p_{N}} T_{q} H g_{N} d x=0 .
$$

By Lemma 2.1, there exists a sequence $\left\{g_{N, j}\right\}_{j \in \mathbb{N}} \subset L^{2}\left(\mathbb{S}^{d-1}\right)$ such that $T_{q} H g_{N, j} \rightarrow u_{N}=e^{x \cdot p_{N}}\left(1+\psi_{N}\left(x, p_{N}\right)\right)$ in $L^{2}\left(B_{R}\right)$, which implies that

$$
\int_{B_{R}}|f|^{2}\left(\prod_{h=1}^{N-1} e^{-x \cdot p_{h}} T_{q} H g_{h}\right)\left(1+\psi\left(x, p_{N}\right)\right) d x=0 .
$$

As $\left|a_{N}\right|=\left|b_{N}\right| \rightarrow \infty$ in (5.35) we have

$$
\int_{B_{R}}|f|^{2} \prod_{h=1}^{N-1} e^{-x \cdot p_{h}} T_{q} H g_{h}=0
$$

Repeating the operation (5.34) $-(5.36) N-1$ times, we have that

$$
\int_{B_{R}}|f|^{2}=0
$$

which conclude that $f=0$. By induction, we conclude that (5.1) for all $l \in \mathbb{N}$. Therefore, Theorem 1.3 has been shown.

\section{Acknowledgments}

The author thanks to Professor Mikko Salo, who supports him in this study, and gives him many comments to improve this paper. 


\section{References}

[1] A. Bukhgeim, Recovering a potential from Cauchy data in the twodimensional case, Journal of Inverse and Ill-posed Problems, 16, 19-33, (2008).

[2] D. Colton, R. Kress, Inverse acoustic and electromagnetic scattering theory, Third edition. Applied Mathematical Sciences, 93, Springer, New York, (2013).

[3] G. Eskin, Lectures on linear partial differential equations , American Mathematical Society, 123, (2011).

[4] A. Feizmohammadi, L. Oksanen, An inverse problem for a semi-linear elliptic equation in Riemannian geometries, Prepreint arXiv:1904.00608, (2019).

[5] M. Harju, V. Serov Three-dimensional Direct and Inverse Scattering for the Schrödinger Equation with a General Nonlinearity, Operator Theory: Advances and Applications, 236, 257-273, (2014).

[6] M. Lassas, T. Liimatainen, Y.-H. Lin, M. Salo, Inverse problems for elliptic equations with power type nonlinearities, Prepreint arXiv:1903.12562, (2019).

[7] M. Lassas, T. Liimatainen, Y.-H. Lin, M. Salo, Partial data inverse problems and simultaneous recovery of boundary and coefficients for semilinear elliptic equations, Prepreint arXiv:1905.02764, (2019).

[8] L. Päivärinta, M. Salo, G. Uhlmann, Inverse scattering for the magnetic Schrödinger operator, J. Funct. Analysis, 259, 1771-1798, (2010).

[9] V. Serov, Inverse fixed energy scattering problem for the generalized nonlinear Schrödinger operator, Inverse Problems, 28, 025002, (2012).

[10] V. Serov, M. Harju, G. Fotopoulosc, Direct and inverse scattering for nonlinear Schrödinger equation in 2D, J. Math. Phys., 53, 123522, (2012).

[11] J. Sylvester, G. Uhlmann, A global uniqueness theorem for an inverse boundary value problem, Annals of mathematics, 125 153-169, (1987).

[12] G. Uhlmann, Electrical impedance tomography and Calderon's problem, Inverse problems, 25, 123011, (2009). 
[13] M. Watanabe, Time-dependent method for non-linear Schrödinger equations in inverse scattering problems, J. Math. Anal. Appl., 459, 932-944, (2018).

[14] R. Weder, Lp-Lp Estimates for the Schrödinger Equation on the Line and Inverse Scattering for the Nonlinear Schrödinger Equation with a Potential, J. Funct. Analysis, 170, 37-68, (2000).

[15] R. Weder, Inverse Scattering for the Nonlinear Schrödinger Equation II. Reconstruction of the Potential and the Nonlinearity in the Multidimensional Case, Proc. Am. Math. Soc., 129, 3637-3645, (2001).

Graduate School of Mathematics, Nagoya University, Japan.

E-mail address: takashi.furuya0101@gmail.com 